\title{
The Use of Wireless Networks for the Surveillance and Control of Vehicles in an Airport Environment
}

\author{
Augusto Casaca ${ }^{1}$, Tiago Silva ${ }^{1}$, António Grilo $^{1}$, Mário Nunes ${ }^{1}$, \\ Franck Presutto ${ }^{2}$, and Isabel Rebelo ${ }^{3}$ \\ ${ }^{1}$ INESC Inovação, R. Alves Redol 9, \\ 1000-029 Lisboa, Portugal \\ \{Augusto.Casaca, Tiago.Silva, Antonio.Grilo, \\ Mario.Nunes \} @inesc.pt \\ ${ }^{2}$ M3 Systems, 1 rue des Oiseaux, \\ 31410 Lavernose-Lacasse, France \\ Presutto@m3systems.net \\ ${ }^{3}$ ANA, Rua D - Edifício 120, Aeroporto de Lisboa \\ 1802-806 Lisboa, Portugal \\ Isabel.Rebelo@ana-aeroportos.pt
}

\begin{abstract}
The paper focusses on the use of wireless networks, with special emphasis on $\mathrm{Wi}-\mathrm{Fi}$, in the manoeuvering and apron areas of an airport to control the ground vehicles movements in those areas and, consequently, to improve user safety, efficiency of operations and airport security. The use of Wi-Fi for these purposes constitutes a novel approach in an airport environment. Other wireless networks, namely CDMA and Tetra, are also experimented in this project as a lower bit-rate alternative to Wi-Fi. The platform consists of an onboard system in each vehicle, a centralised ground system and wireless networks to allow the communication between the vehicles and ground system. The architecture, protocols and network configurations in use are analysed as well as the respective deployment made in the airport of Porto in Portugal.
\end{abstract}

\section{Introduction}

The continuous and steady growth of air traffic leads to an escalating number of accidents and incidents on surface movements. In case of low visibility, since the surveillance and control of movements are based mostly on the "see and be seen" principle, airport stakeholders have little knowledge of ground surface traffic, thus leading to ground movement hazards. In addition, airport congestion is also becoming an increasing problem.

The AIRNET (AIRport NETwork for Mobiles Surveillance and Alerting) project ${ }^{1}$ has the high level objectives of improving user safety, efficiency of operations and airport security in the apron and manoeuvering areas of an airport.

The project developed a GPS/EGNOS based low cost platform for the surveillance, control and management of all airport vehicles (eg. catering, luggage, fuel,

\footnotetext{
${ }^{1}$ The AIRNET project is partially funded by the European Commission in the sixth Framework Program under contract $\mathrm{n}^{\circ} 507888$.
} 
maintenance, police, firebrigade, etc). These services implement the recommendations of Eurocontrol for A-SMGCS (Advanced Surface Movement Guidance and Control Systems) [1]. The platform consists of an on-board system in each vehicle, a centralised ground station and wireless communication networks to interconnect all the systems [2] [3].

Concerning the wireless communication networks used for this solution the main emphasis is put in the use of Wi-Fi, which is a complete novel approach for these types of applications in an airport environment. In parallel, CDMA and Tetra technologies will also be experimented so that conclusions can be taken on the use of technologies with a lower bit rate than Wi-Fi for the applications envisaged. Finally, for a limited number of situations, a VDL-4 network is also experimented to demonstrate the ability of the AIRNET platform to be compliant with one aeronautical network.

The AIRNET project lasts for three years and is presently running in its last year, in which the AIRNET platform is being deployed in the Porto airport in Portugal, which can be considered a medium sized airport. The platform and the use of the wireless communication networks will be validated by running a complete set of operational scenarios, which have been established by the airport stakeholders. The AIRNET platform is expected to be part of future airport management systems as a building block that can be integrated in A-SMGCS.

In the next section of the paper the AIRNET services will be shortly presented. Section 3 will introduce the AIRNET platform components. Sections 4, 5 and 6 are dedicated to the communication network architecture and to the communication technologies experimented. Section 7 describes the network deployment and finally conclusions are drawn in the last section of the paper.

\section{Airnet Services}

The AIRNET services will help actors to improve safety for the vehicle movements in the manoeuvering and apron areas of the airport [4]. The AIRNET actors are the Air Traffic Controller (ATCO), the Airport Operation Officer (AOO), the Ground Handling Manager (GHM) and the vehicle drivers.

AIRNET provides four types of services: surveillance, control, guidance and decision support.

The surveillance service dedicated to the ATCO provides continuous surveillance data for the manoeuvering area. The data consists of traffic information on all the aircraft and vehicles in the area and traffic context, which is the airport map representation. The same service is provided to the remaining actors by extending it to the apron area too.

The control service dedicated to the ATCO aims to provide conflict/infringement alerts for the manoeuvering area. It detects conflicts/infrigements on runway caused by aircrafts or vehicles, on taxiway when a vehicle crosses a taxiway while an aircraft is taxing and by incursions in restricted areas. The same service is provided to the 
remaining actors, but including the apron area by detecting conflicts betwwen two vehicles or an aircraft and a vehicle.

The guidance service is dedicated to vehicle drivers and includes all the areas of the airport. This service allows drivers to visualize their own positions on a moving map of the airport.

The decision support service is dedicated to the drivers, AOO and GHM. This service aims to provide situation assessment and solutions to optimize the use of vehicles. The decision support service, in concrete, provides real time information about aircrafts, text messages with instructions for the drivers, historical data of the airport and vehicle status information.

\section{Airnet Platform}

In order to provide the proposed services, the following key enablers are required: human-machine interfaces for the vehicle drivers and ground system users, mobile postioning using GPS/EGNOS, data flow between the vehicles and the central ground system, processing units for running the software applications that provide services to drivers and ground system users.

The AIRNET platform integrates all these enablers and its architecture includes three components: on-board systems, ground system and communication networks. This architecture is shown in Fig. 1.

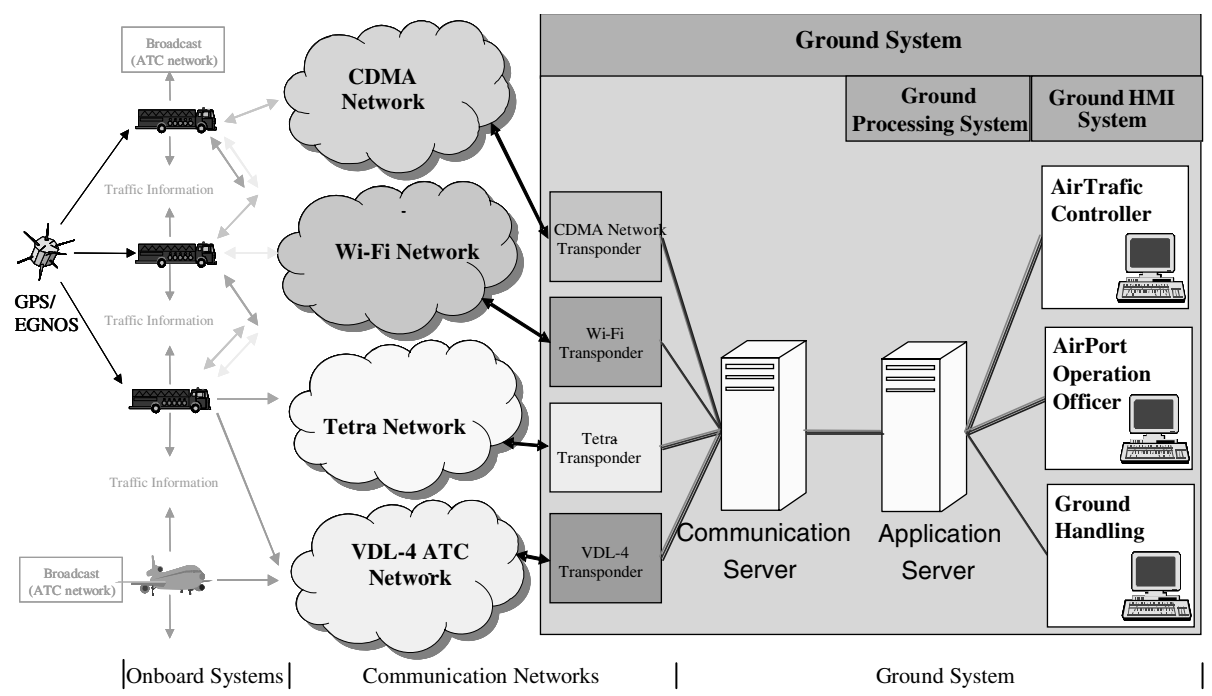

Fig. 1. Architecture of the AIRNET platform

There is one on-board system installed in each vehicle. The system consists of a Communication and Navigation Unit (CNU) and an on-board display for the vehicle driver. The CNU includes a GPS/EGNOS receiver, the wireless communication 
network transponders and a PC board. The software modules that implement the AIRNET services on-board run in the PC board. There are five distinct software modules, which are identified according to the services that they provision, namely Traffic Information, Traffic Context, Conflict/Infringement Detection, Service Monitoring (monitors the equipment status) and Decision Support.

The ground system consists of the application server, communication server with the network transponders and a set of monitors for the users of the platform. Software modules equivalent to the ones running in the $\mathrm{CNU}$ are running in the application server (ground processing system), namely Traffic Information, Traffic Context, Conflict/Infringement Detection, Service Monitoring and Decision Support. The ATCO, AOO and GHM interact with the platform via the monitors (ground HMI) connected to the application server. The communication server concentrates all the software tasks concerned with the management of the communication links to the on-board systems.

The communication networks allow the exchange of data between the on-board systems and the ground system. They cover the whole maneuvering and apron areas of the airport. Three distinct networks are used for this purpose: Wi-Fi, CDMA and Tetra. The main emphasis of the project is on the use of Wi-Fi, which is a novel approach for an airport maneuvering area environment and, therefore, this network by itself is enough to satisfy all the requirements of the AIRNET platform. However, the project intends also to demonstrate that the platform can also be implemented, although with a lower quality of service, using lower bit-rate communication technologies like CDMA and Tetra. These two networks are commonly found in an airport environment and that is the main reason of their choice as alternative technologies to Wi-Fi. Finally, the VDL-4 network is only used to demonstrate that some of the services can also be implemented in an aeronautical network. The communications server (CS) is the entity responsible for managing the heterogeneity of the wireless communication networks, presenting a common network interface to the ground system services. Like the CNU, its implementation is also based on a PC architecture running the LINUX OS. In the remaining of the paper we only concentrate on Wi-Fi, CDMA and Tetra, which are the networks where all the services can run.

\section{Airnet Network Architecture}

The detailed AIRNET network architecture is depicted in Fig. 2, where the interconnection to the airport's LAN is also shown. The AIRNET communications are completely based on the IP protocol. The core of the AIRNET network consists of an interconnection VLAN that is separate from the airport LAN where the Airport Operational Management system (AOMS) is located. The Wi-Fi network forms itself an independent VLAN that connects to the AIRNET VLAN.

Within the AIRNET system the details of the communication network are completely transparent to the services. Higher layer software modules, including application modules running on both the CNU and the application server exchange service messages in ASTERIX [5] format without knowing the medium through which the messages will effectively be transmitted. Networking details are dealt with by the Communication Server (CS) in the ground system as referred previously. 


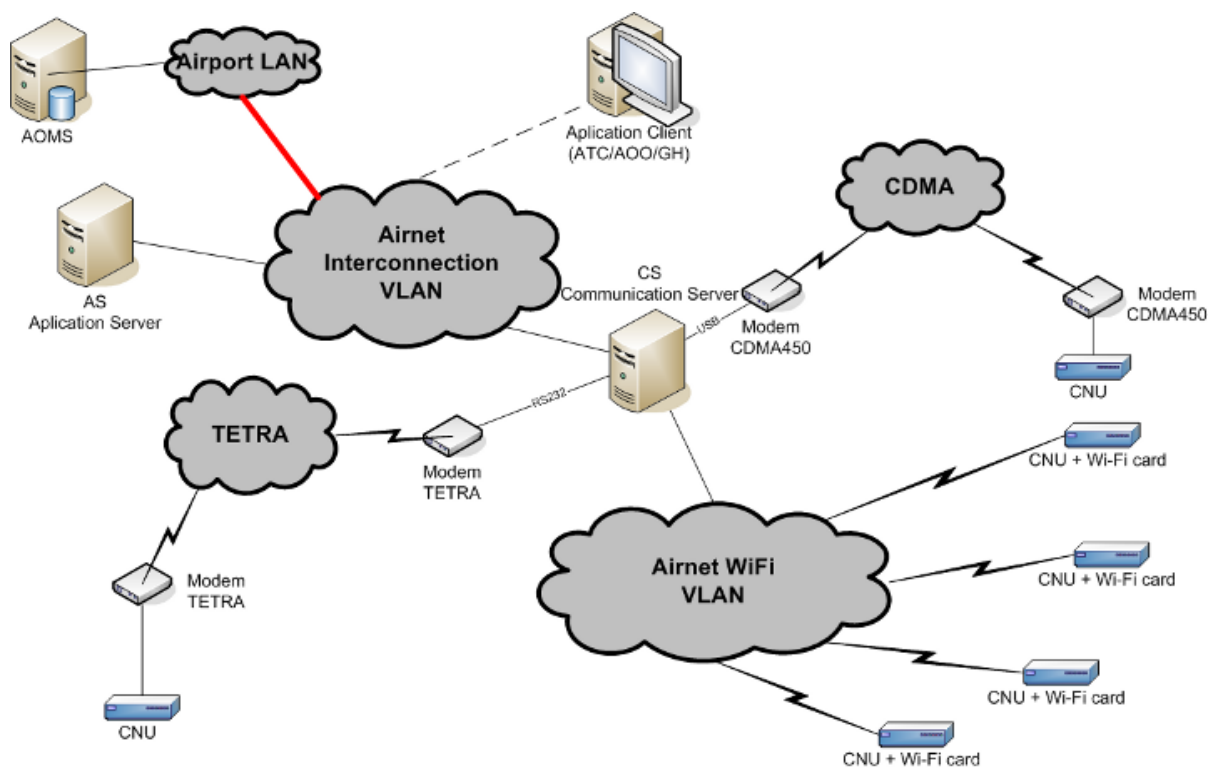

Fig. 2. AIRNET network architecture

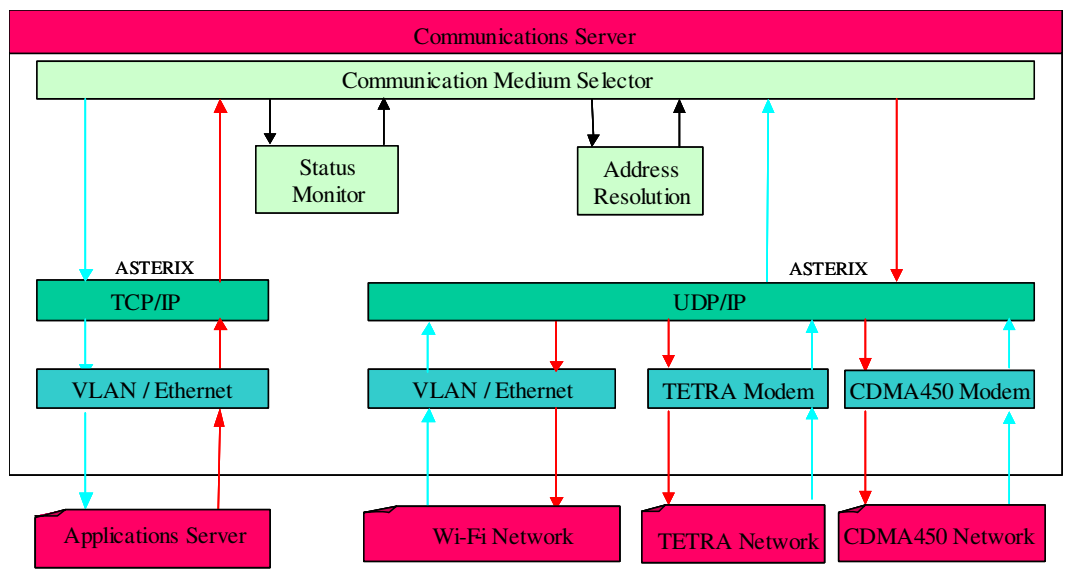

Fig. 3. Architecture of the communication server

Within the CS, the Communication Medium Selector is responsible for the routing decisions, choosing between different wireless technologies. The CNUs and CS are the endpoints of communication from the perspective of the transport layer. Due to the unsuitability of TCP to operate over the air interfaces, communication between the CNUs and the CS is based on the UDP transport protocol. For the services that require stringent delivery guarantees, reliability is implemented at the application layer. Within the cabled network that supports the ground system, TCP is used for added reliability. The gateway between UDP and TCP is located at the communications server. 
ASTERIX messages issued by the onboard systems to other onboard systems or to the ground system, as well as messages issued by the application server to the onboard systems are all routed by the CS. Independently of the source, broadcast messages are sent in unicast mode to the CS, which then activates the broadcast mechanisms supported by the target air interface (e.g., broadcast transmission for Wi-Fi and pseudo-broadcast - i.e. multiple unicast - transmission for Tetra and CDMA).

ASTERIX message destinations are uniquely identified by a specific 24-bit Target Address field. Address translation between Target Address codes and UDP/IP addresses is performed at the CS. Another networking function performed by the CS has to do with network transponder status monitoring. The CS periodically checks the status of the network transponders by means of IP-based Ping requests and any failures are readily reported to the general Service Monitoring module at the applicationsserver by means of special ASTERIX messages using a separate TCP port.

The three wireless networks that integrate the AIRNET platform will now be described in greater detail.

\section{Tetra and CDMA Networks}

\subsection{The Tetra Network Architecture}

The motivation to test the use of Tetra is that a Tetra network is already deployed at the Porto airport to support voice services and it has the capability of supporting data services too.

The TETRA network is centered on a base station (BS) and supports packet data services with the Specific Connectionless Network Service (S-CLNS). The S-CLNS allows the transmission of IP packets between a TETRA mobile terminal and terminals located in either a fixed IP-based LAN or other mobile terminals using the SCLNS (see Fig. 4). In AIRNET, the proprietary DIMETRA S-CLNS implementation

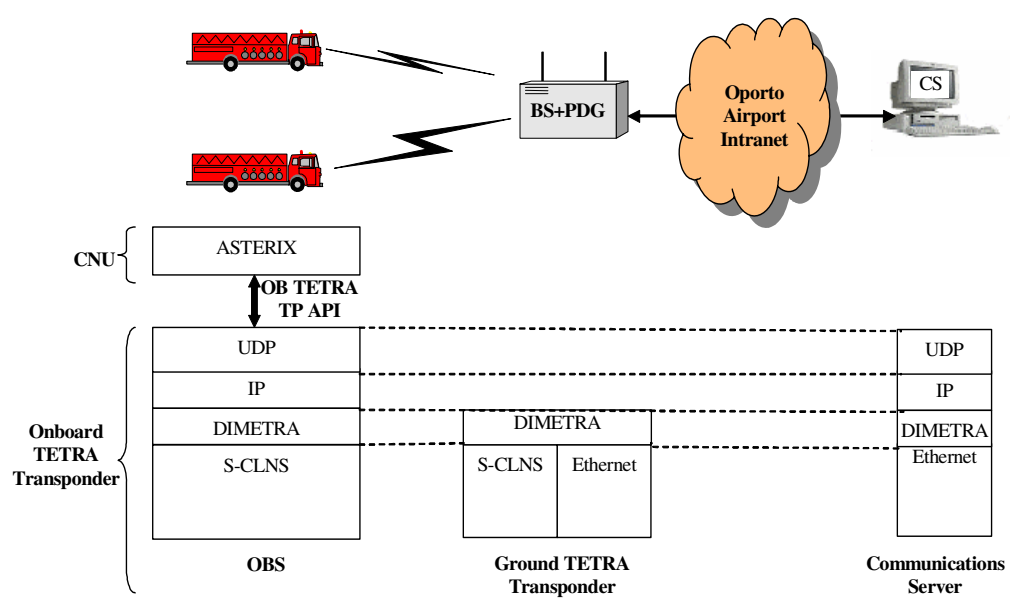

Fig. 4. Architecture of protocols in the Tetra network 
is used [6]. The interface with the IP-based LAN is performed by a PC running the Packet Data Gateway (PDG) software. This equipment, together with the BS forms the logical Ground TETRA Transponder when the S-CLNS service is in use. UDP was again chosen as the transport protocol due to the reasons already mentioned for Wi-Fi.

Due to the low bitrate supported by the Tetra technology (maximum of $28.8 \mathrm{kbps}$ per carrier per direction of communication) and the overhead introduced by UDP/IP communication, the AIRNET functionality will be reduced when this technology is used.

\subsection{The CDMA Network Architecture}

The CDMA network available in Portugal operates in the $450 \mathrm{MHz}$ range. It basically follows the specifications of CDMA2000 defined by 3GPP2, with the physical layer modifications required for use in another frequency band and with lower bandwidth channels (1.25 MHz).

The Portuguese CDMA network operates in two modes: 1xRTT (Radio Transmission Technology) [7] and EV-DO (Evolution, Data Optimized) [8].

In the EV-DO network the maximum bandwidth per sector is $2.4 \mathrm{Mbps}$ downstream and $153.6 \mathrm{Kbps}$ upstream. In the 1xRTT mode the maximum bandwidth per sector and connection is $153.6 \mathrm{Kbps}$ for both downstream and upstream directions. Presently the EV-DO is only available in urban and suburban areas, what fortunately includes the entire AIRNET platform at the Porto airport, requiring, however, that the ground vehicles are equipped with external antennas.

The protocol architecture of the CDMA450 is shown in figure 5.

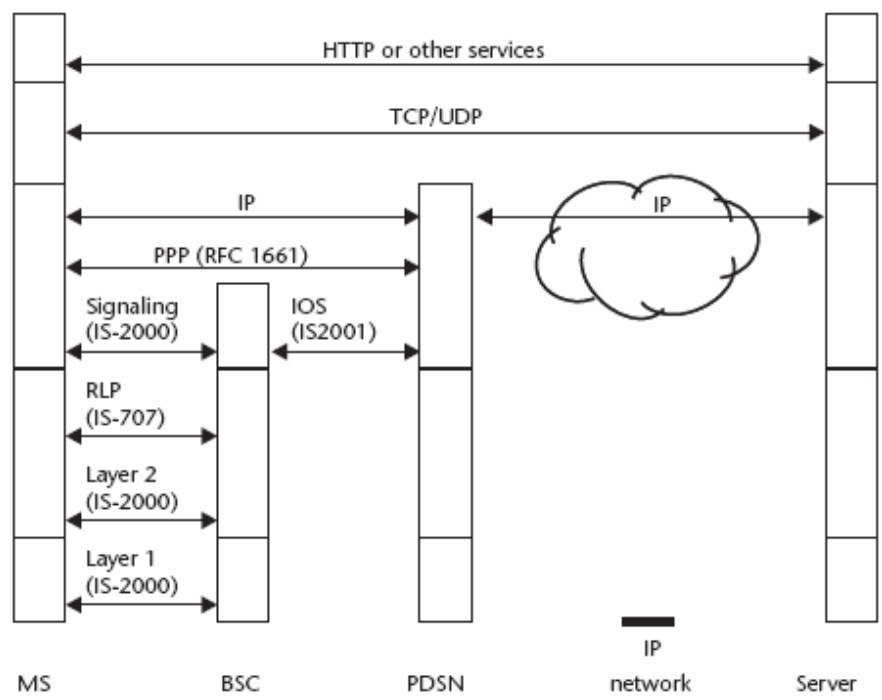

Fig. 5. Architecture of protocols in the CDMA network 
On of the advantages of the CDMA protocol architecture is that it is IP based, so its integration with the Airnet network subsystems is seamless and efficient.

Another advantage of CDMA for the Airnet stakeholders is that besides allowing high speed data, this network also provides voice services, both inside the network and also to other public networks, fixed and mobile. Consequently it enables the airport users to establish and receive telephony calls with any telephone user of any network, as well as to access all "normal" telephony call services that mobile telephone users are used to.

Still another important advantage of CDMA450 is that as it operates in a frequency band much lower than UMTS or Wi-FI, it has a much lower attenuation in open air and better coverage in non line-of-sight environments, what means that it can cover a wide area with a single Base Station Controller, making it a possible alternative to support the Airnet services.

\section{The Wi-Fi Network Architecture}

AIRNET gave special emphasis to the use of Wi-Fi to support the AIRNET services due to its high data rate when compared with other networks, namely Tetra and even CDMA. Besides, Wi-Fi is a private network totally controlled by the airport authorities. This does not happen with CDMA, which is a network under control of a public operator.

In the AIRNET platform the Wi-Fi network is based on the IEEE 802.11a standard [9], which operates in the $5 \mathrm{GHz}$ frequency band, supporting physical bit-rates between $1 \mathrm{Mbps}$ and $54 \mathrm{Mbps}$. IEEE 802.11a was selected in detriment of its $2.4 \mathrm{GHz}$ counterpart standard IEEE 802.11g [10] due to the fact that the Portuguese communications regulations authority (ANACOM) has authorized the use of higher transmission power in the $5.470-5.725 \mathrm{GHz}$ frequency band for vehicular applications (1 W E.I.R.P versus $100 \mathrm{~mW}$ E.I.R.P for the $2.4 \mathrm{GHz}$ frequency band), which greatly reduced the number of APs required to cover the airport.

Among the analysed Wi-Fi architectures, the IP-based Routed-WLAN architecture turned out to be the most advantageous. The Routed-WLAN architecture uses high layer management protocols on top of TCP/IP to configure the network elements: Central Controller and Access Points (AP).

The Central Controller concentrates all the Wi-Fi network "intelligence". Its main responsibilities are:

- Network management services.

- RF channel management.

- AP transmission power management that automatically adapts to interference conditions.

- Handover logic and mobility management.

- Enforcement of security and QoS policies.

- VLAN/VPN management.

- Network monitoring and automatic reconfiguration in response to failure conditions.

The APs have their functionality limited to the PHY and MAC layers, except for the management plane. 
In the AIRNET architecture, the APs and the Central Controller belong to a separate VLAN that interconnects to the CS through the interconnection VLAN (see Fig. 2). The main advantages of the selected Routed-WLAN architecture are the following:

- Security: Support of IEEE 802.11i [11] features and Virtual Private Networks.

- Efficiency: Handover between APs in the same IP subnetwork has a lower latency, since it is processed in the same Central Controller. Authentication is only required the first time that the mobile terminal associates to the WLAN.

- Compatibility: Although the full set of advantages provided by the RoutedWLAN requires specific functionality at the APs, any AP can be attached to the network.

- Scalability: The Central Controller is the most complex component in the architecture, but it can control hundreds of APs and can be located anywhere in the IP network. Unlike in traditional WLANs the network administrator is able to manage the complete network at this single point, instead of separately configuring each AP.

- Reliability: Network redundancy can be easily obtained through the addition of extra Central Controllers that can automatically take control in case of failure of the operating Central Controller. Likewise, in case of failure of one AP, other APs covering the same area can be automatically re-configured to take control of traffic. The Central Controller also implements automatic power control mechanisms to compensate for external interference and/or janmming.

- Virtualization: The traffic generated by different airport actors (e.g. ATC, AOO, GHM) can be isolated and separately managed based on network access and utilization policies, including QoS.

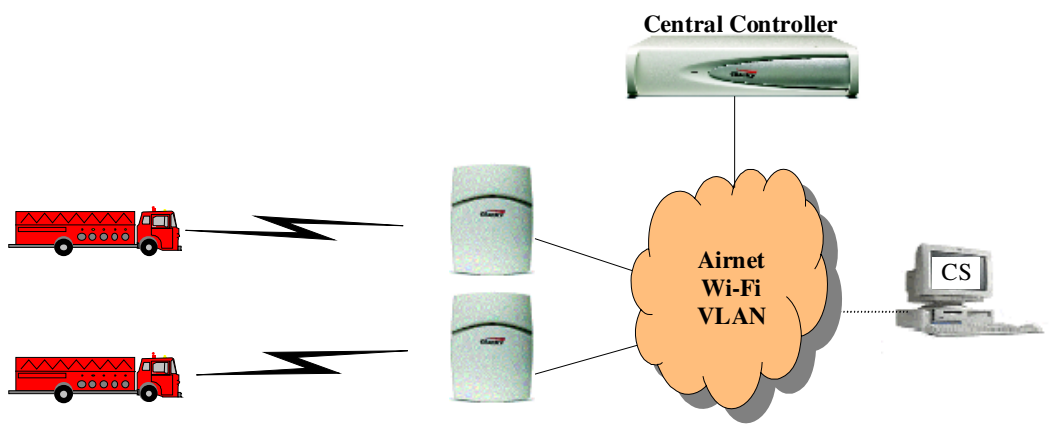

\begin{tabular}{|c|c|c|c|}
\hline UDP & & & UDP \\
\hline IP & & & IP \\
\hline VLAN & Wi-Fi & Wi-Fi & Ethernet \\
\cline { 1 - 1 } $\begin{array}{l}\text { Onboard Wi-Fi } \\
\text { Transponder }\end{array}$ & \multicolumn{1}{|c|}{$\begin{array}{c}\text { Ground Wi-Fi } \\
\text { Transponder }\end{array}$} & Ethernet \\
\hline
\end{tabular}

Fig. 6. Architecture of protocols in the Wi-Fi network 


\section{Deployment}

There are currently three deployed wireless networks as part of the AIRNET system: TETRA, CDMA450 and WiFi (802.11a) besides the aeronautical VDL-4 network, which is only used for some services as indicated earlier. Tests on the use of these networks to support the AIRNET services have already started.

The Tetra network covers the entire airport, and most of the airport staff vehicles were already equipped before with Tetra for voice and short message communications. Preliminary results of the data transmission tests in the Tetra network within the AIRNET platform show a limited performance for the AIRNET services. These results point to the need of reducing the number of AIRNET services based on Tetra transmission.

The CDMA network also covers the complete airport area, using ZAPP Telemodem Z010 inside the vehicles, even without external antenna. The airport area is covered in CDMA/1xRTT, and one half of that area is also covered with CDMA/EVDO. Preliminary tests show that CDMA performance is satisfactory for the AIRNET requirements.

The Wi-Fi (IEEE 802.11a) network deployment was intended to assure the complete coverage of the Porto airport apron and manoeuvering areas. The increased transmit power allowed for IEEE 802.11a allowed this goal to be achieved with only 17 APs, which are also able to provide coverage redundancy (see Fig 7).

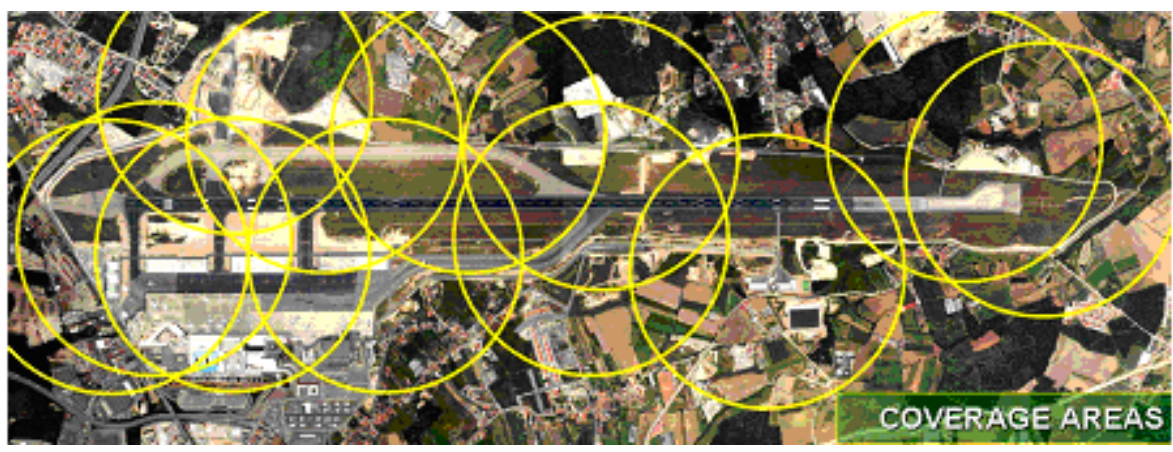

Fig. 7. Distribution of APs and Wi-Fi (802.11a) coverage at the Porto Airport

Preliminary tests show that on the average, each AP is able to cover from 0 to $\sim 1$ $\mathrm{Km}$ (vehicle using external antenna and running at $40 \mathrm{Km} / \mathrm{h}$ ), with performance going far beyond AIRNET requirements, which allow future support of other services like, for example, EGNOS broadcast, VoIP (e.g., Wi-Fi Phone) and video streaming,

\section{Conclusions}

The AIRNET communication networks are already deployed in the Porto airport covering the entire apron and manoeuvering areas. The remaining parts of the 
AIRNET platform are also in deployment and initial tests with a limited number of vehicles have started. The tests are part of a set of operational scenarios, which have been defined by the airport stakeholders. They include different pratctical situations, such as, aircraft from landing to stand, aircraft from stand to take-off, runway inspection by the air traffic control service vehicle, conflict on the runway involving a vehicle and an arriving aircraft, conflict in the apron involving a vehicle and an arriving aircraft and ground handling management.

The results obtained until now show the usefulness of the AIRNET services for an efficient control of the airport vehicles and show also that CDMA and Wi-Fi are networks fully capable of supporting this type of applications. Wi-Fi has the advantage that due to its higher data rate can easily support a wider range of new applications in this environment.

\section{References}

1. International Civil Aviation Organization, European Manual on Advanced Surface Movement Control and Guidance Systems (A-SMGCS), Doc 9830 AN/452, 2004.

2. Casaca, A., Presutto, F., Rebelo, I., Pestana, G. and Grilo, A., Na airport Network for Mobiles Surveillance, in Proc. of the $16^{\text {th }}$ International Conference on Computer Communications, ISBN 7-121-00308-2, pp. 1703-1708, Beijing, China, 2004.

3. Grilo, A., Nunes, M., Casaca, A., Presutto, F., Rebelo, I., Communication Network Architecture for Mobiles Surveillance in an Airport Environment, Joint International Symposium on Sensors and Systems for Airport Surveillance Proceedings (CDROM), Paris, France, 2005.

4. AIRNET Deliverable D1.1, AIRNET Operational and Systems Requirements, AIRNET/D1.1/M3S/WP1/OP_SYS_REQ/2.0, http://www.airnet-project.com/, July 2004.

5. Eurocontrol, "EUROCONTROL Standard Document for Surveillance Data Exchange, Part1: All Purposed Structured Eurocontrol Surveillance Information Exchange (ASTERIX)", v 1.29, February 2002.

6. Motorola, "DIMETRA Packet Data Service - Programmer's Guide", Release 3.1, March 2000.

7. S. Agrawal, I. Acharya, S. Goel, "Inside 3G Wireless Systems: The 1xEV-DV Technology"; TATA Consulting Services, March 2003

8. Lawrence Harte, "Introduction to EVDO, Physical Channels, Logical Channels, Network and Operation"; Althos Publishing, 2004

9. IEEE 802.11a, "IEEE Standard for Telecommunications and Information Exchange Between Systems - LAN/MAN Specific Requirements - Part 11: Wireless Medium Access Control (MAC) and physical layer (PHY) specifications: High Speed Physical Layer in the 5 GHz band", IEEE, 2003.

10. IEEE 802.11g, "Wireless LAN Media Access Control (MAC) and Physical Layer (PHY), Specifications: Higher-speed Physical Layer (PHY) Extension to IEEE 802.11b", IEEE, 2003.

11. IEEE 802.11i, "Wireless Medium Access Control (MAC) and physical layer (PHY) specifications: Medium Access Control (MAC) Security Enhancements", Draft version 10, IEEE, 2004. 